\title{
LA PSICOLOGÍA SOCIAL: ENTRE LA ACCIÓN SOCIAL COMPROMETIDA Y EL ASISTENCIALISMO
}

\author{
Rousseau decía: "Una sociedad sólo es democrática cuando \\ nadie es tan pobre para tener que venderse y nadie tan rico \\ para poder comprar a alguien" Algo muy diferente de lo que \\ ocurre hoy en día... cit. Por Boaventura de Souza Santos
}

\author{
CARlos ArenAs IPARRAguire $^{1}$ \\ Universidad Nacional Mayor de San Marcos, Perú \\ (Recibido el 03/09/2007, aceptado el 30/10/2007)
}

\section{PRESENTACIÓN}

Es necesario empezar con las cifras demostrativas del mundo actual: hoy, un $20 \%$ de los seres humanos que viven en los países más ricos consumen un $86 \%$ de los bienes producidos por la humanidad, y el $20 \%$ de la población mundial, que vive en los países más pobres, no consume más de $1,3 \%$ del total, mención aparte y que desnuda mucho más la situación de distribución desigual de la calidad de vida es considerar las distintas cifras del denominado Índice de Desarrollo Humano elaborado por el Programa de las Naciones Unidas para el Desarrollo - PNUD.

El abordaje de problemáticas de la pobreza y sus efectos perniciosos en todos los ámbitos del "mundo de la vida" tales como el desempleo, la marginalidad, la exclusión social, particularmente para los sectores que se denominan pobres y/o pobres extremos, efectos de los cuales no escapan muchos sectores de las clases medias, particularmente las tradicionales en proceso de descenso a los niveles de pobreza.

No es indiferente la problemática generada por los procesos de modernización exógena impulsadas por dinámicas generadas por la mundialización y los nuevos términos de intercambio entre las nuevas políticas coloniales y los procesos de colonización interna, no ajena al papel desempeñado por las clases dominantes de nuestros diversos países, reproducido por las élites regionales y locales, particularmente de lo que se ha denominado provincias del "interior" del Perú. Entendemos que no hay que ubicarse en la perspectiva ni antropocéntrico-metropolicéntrica ni entender que hay una polaridad para el entendimiento de los fenómenos: estos son complejos y cambiantes, en la que la acción humana le corresponde el rol de reproducir los cambios organizacionales e institucionales, en función

1 Docente Principal de la Facultad de Psicología de la UNMSM. E-mail: cardenasi@unmsm.edu.pe 
de intereses definidos, tanto individuales, grupales, comunitarios, locales, regionales o nacionales.

Es dentro de esta perspectiva que habría que incluir la reflexión desarrollada por Castoriadis, C. (1993) sobre el mundo subjetivo, foco de atención prioritaria de la disciplina psicológica, observatorio desde el que desarrollamos el presente acercamiento. Este autor señala que las sociedades occidentales se conformaron por la articulación de dos imaginarios sociales contrarios: el imaginario social capitalista y el imaginario social democrático.

El imaginario social capitalista se caracteriza por la competitividad, el objetivo de máxima productividad, y de obtención del máximo beneficio. Este imaginario promueve un esquema referencial o mentalidad capitalista. El ideal es una subjetividad competitiva, exitosa, individualista, que se rige por una lógica de guerra simbólica, centrado en la producción y en el enriquecimiento personal desentendiéndose si las consecuencias de ello implica la exclusión social de sus conciudadanos, por otro lado le corresponde al imaginario social democrático promover valores opuestos al capitalista. Promueve ideales relacionados con el bien común, la solidaridad, la convivencia con el diferente. El diferente no es alguien sobre el cual triunfar sino el que aporta por ser, justamente, diferente. Aunque él no era futurólogo afirma que el imaginario social capitalista ha avanzando sobre el democrático en áreas que antes eran impensables como el arte o la justicia, para ello es interesante contrastar con reflexiones de autores representativos como el sociólogo Giddens, A. o el historiador Hobsbawm, E., o desde la economía lo hace Sen, A.

Nuestra sociedad actual se halla fragmentada en numerosos grupos sociales que poseen diversos intereses y opiniones y que imposibilitan un referente común que permita el diálogo y el disenso aumentan simultáneamente la desigualdad interior y la existente entre naciones.

Sobre la base de lo sustentado, en relación a la mundialización y la nueva colonización (externa e interna), se podría confirmar algo como la pérdida de una razón globalizante la cual ha hecho emerger racionalidades específicas que operan muchas veces de manera cerrada y autónoma. La cultura, la economía, la política, la ciencia, los espacios geopolíticos, las propias instituciones operan según lógicas autorreferenciales que hacen difícil un consenso común. Esta situación vuelve difícil construir un imaginario social colectivo que pueda mantener unida a la sociedad dando una noción clara de sí misma.

Las grandes ciudades han perdido su centro. Ya no hay un centro de la ciudad. Toda gran ciudad tiene múltiples centros. Parecería que las metrópolis son una metáfora de la sociedad. La sociedad también ha perdido su centro. No existe un referente común para los diversos grupos sociales, particularmente para los sectores mayoritarios que se ubican en los márgenes de lo económico-social. La globalización ha generado además un redimensionamiento del espacio. Cada campo, en términos de P. Bourdieu (1995), puede trascender los límites de su país. De hecho comparten sus problemáticas y potencian sus recursos más allá de sus fronteras nacionales al mismo tiempo que se alejan de las lógicas de los otros campos de su propio país. En ese entresijo, las distancias entre los diferentes países, grupos y clases sociales a la vez que se estrechan también aumentan la distancia entre grupos sociales dentro de un mismo país. 
De esta forma los esquemas de referencia, las teorías implícitas: la subjetividad se van conformando a partir de complejas tramas vinculares que se distinguen en ámbitos individuales, grupales, institucionales y comunitarios. Este heterogéneo proceso de socialización da lugar en la subjetividad a la asunción de determinados modelos de pensar, sentir y hacer en el mundo: la modernización que conlleva la construcción, entre otros, del individuo moderno traía aparejada una fragmentación de los esquemas referenciales

La fragmentación del esquema referencial, de la identidad personal-social, produce incertidumbre, angustia, pérdida de identidad. Se pierden las claves de interpretación de la realidad y de anclaje emocional. Y esto afecta en mucho mayor grado a los sectores de menores recursos. Menores recursos no solo económicos, sino de estímulos intelectuales, de información, de recursos de legitimidad de sus derechos etc. Esta fragmentación tiene un peligro que le es inherente, el sujeto en situación de incertidumbre, de angustia tiende a tomar medidas extremas, autoritarias, de desesperanza, de recelo y resentimiento, de fragmentación de su propio ambiente y las redes sociales que lo sustentan. Tiende además a descreer de la tolerancia, la libertad, en síntesis, de la democracia.

La Psicología Social es la disciplina -interdisciplina en rigor- capaz de potenciar los recursos y la cooperación de subjetividades, grupos y organizaciones tendiente a la constitución de redes porque cuenta con metodologías y técnicas participativas orientadas a un objetivo de transformación social.

Desde una preocupación inicial por los procesos colectivos y los grupos sociales se pasó a poner el énfasis en la interacción y, finalmente, en los procesos psicológicos individuales, es decir en procesos psicológicos internos.

En la mayor parte de la investigación sobre los procesos de atribución causal y, particularmente, en los estudios sobre "sesgos" atribucionales, éstos son analizados como si fueran procesos de carácter interno. Además, al ser entendidos como principios de carácter universal, se ignora, necesariamente, el contexto social

Tampoco es posible introducir los procesos afectivos que influyen en el conocimiento que tenemos de la realidad. Existen, lógicamente, excepciones como las de Hewstone, quien intenta dar un enfoque más social de las atribuciones. Los estudios sobre cognición social podrían perfectamente rescatar el enfoque del interaccionismo simbólico, o los estudios de Vygotsky, Luria, Leontiev, etc.

Los estudios sobre cognición no han tenido en cuenta ni el papel del lenguaje ni la influencia de la interacción social. Se ha ignorado que la interacción simbólica es la base en la construcción de la conciencia. Es apropiada la propuesta de un psicólogo social como McGuire quien aboga por un contextualismo teórico en el que se defiende que no hay teorías verdaderas o falsas, per se, sino que el potencial explicativo o interpretativo de cada teoría depende del objeto de estudio.

Lo que necesitamos no es tanto una ciencia unificada como una mayor contextualización del conocimiento, así como un mayor pluralismo teórico. 


\section{¿PSICOLOGÍA COMO CIENCIA SOCIAL UNIFICADA?}

Tanto desde el punto de vista teórico como metodológico, se debería ir más hacia una complementariedad e interdisciplinariedad que hacia la idea de una ciencia social unificada.

Articular las diferentes perspectivas y al mismo tiempo tener en cuenta una perspectiva en la psicología social, en donde realmente se considere su carácter histórico y contextual: como Ignacio Martín-Baró, él tenía un amplio conocimiento de la psicología social, pero al mismo tiempo siempre tuvo en cuenta la realidad histórica a la que iba a aplicar ese conocimiento; es decir, las condiciones históricas de El Salvador en las que él estaba viviendo.

No existe una psicología social o un conocimiento psicosocial particularizado por contextos geográficos, Sí sería posible una psicología social que surge y se desarrolla en respuesta a la problemática de esos contextos geográficos diferentes.

Existe un paralelismo, a veces conflictivo, de las teorías y la producción teórica académica universitaria, con las intervenciones psicosociales en problemas sociales y de salud pública.

\section{COMPLEJIDAD DE DEFINIR LA PSICOLOGÍA SOCIAL, SU OBJETO, SU ROL Y SU HISTORIA}

Esta complejidad que se encuentra atravesada por estas tradiciones y contradicciones, que tienen enormes implicancias a la hora no sólo de aproximarse teóricamente, sino de desarrollar estrategias de intervención, y pensar desde qué "lugares" pueden ser realizadas.

Existen principios de la psicología que pueden tener algunas dimensiones de carácter universal (etic.), propias de la dimensión científico académica. En esta perspectiva Rappaport (1977) planteó considerarla como una perspectiva orientada a la búsqueda de nuevos paradigmas, o nuevas formas de comprender y actuar, las cuales debían considerar tres elementos importantes: a) la relatividad cultural; b) la diversidad humana y el derecho de la gente a acceder a los recursos de la sociedad y elegir sus metas y estilo de vida y c) la ecología o ajuste entre personas y ambiente en el que se destaca el social como un determinante del bienestar humano.

Otros autores como Mead o Vygotsky, que vincularon los procesos cognitivos a la producción social de significados indicaron las rutas por las cuales sólo así es posible un estudio de la mente en el que no queden excluidos los procesos simbólicos.

Progresivamente se han ido mezclando tradiciones académicas y prácticas sociales, cuyo eje aglutinador han sido los problemas sociales.

Existe una demanda teórico-técnica a la psicología social en torno a los problemas sociales existentes. Y es desde allí donde han surgido o se han expresado el aporte de distintas categorías psicosociales (Germán Morales F.). 
Según Sergei Moscovici, Enrique Pichon-Riviére e Ignacio Martín-Baró, hay necesidad de revisar y teorizar sobre las prácticas sociales y los problemas que se derivan de ella. Ellos apelan a un desafío descriptivo, comprensivo y crítico de los problemas sociales desde la psicología, donde cada vez más la subjetividad como concepto genérico se vislumbra como eje del análisis de lo que los sociólogos llaman temas "emergentes".

A la rigurosidad que implica el carácter científico de la Psicología se incluye el criterio de "relevancia social". Pero relevancia social no sólo entendiendo por ello que el objeto de estudio sea socialmente relevante, no cabe duda de que, por ejemplo, el desempleo lo es, sino también que resulte relevante el conocimiento sustantivo que se obtiene de las investigaciones.

\section{ARTICULACIÓN CON LA NUEVA MUNDIALIZACIÓN O GLOBALIZACIÓN}

Estos temas emergentes se ponen sobre el tapete en polémicas ya cotidianas sobre la seguridad ciudadana y la delincuencia, la corrupción, la violencia intrafamiliar, la sexualidad y el SIDA / VIH, y en especial sobre la llamada "superación de la pobreza".

Los grandes problemas que enfrentan la América mestiza, en el marco del proceso de globalización, y que requieren del aporte desde la ciencia, la academia, la profesión y la investigación psicosocial serían, entre otros: los espacios urbanos y rurales, la sustentabilidad, la interculturalidad, los Derechos de las minorías discriminadas, Género, la violencia social y doméstica, las adicciones, no solo a las sustancias psicoactivas sino a las nuevas formas de adicción, la exclusión y marginación social, etc.

A las seculares condiciones de desigualdad, pobreza y exclusión que han acompañado la historia de América Latina, se han sumado nuevos e inéditos fenómenos que reproducen el circuito perverso de empobrecimiento material y espiritual de las personas y las sociedades. A los 'viejos pobres' se les unen ahora jóvenes precariamente empleados o directamente excluidos con más escolaridad y más frustración muchos de los cuales, y como parte del "drain-brain" emigran como profesionales a otras regiones del mundo 'rico', desangrando nuestro países.

Se precisa ampliar la noción de los derechos humanos en el sentido de los derechos colectivos, como los de las mujeres e indígenas. La cuestión indígena es una de las luchas más emancipatorias del continente americano, que nos va a convocar a pensar la autodeterminación de una nueva manera. Un reto para la Psicología Social es acercarse a los movimientos como el Foro Social Mundial, el precedente se realizó en Porto Alegre (2001), representó un marco: fue el primero del Hemisferio Sur, en un país "en desarrollo", organizado no contra algo, sino a favor de una alternativa", lo mismo corresponde a incluir en su agenda el tema del desarrollo sustentable y la Agenda 21.

La localización de la globalización involucra el reto hacia una política de la identidad y la diferencia. Dentro de la globalización se están forjando nuevas identidades donde se conjugan diferentes regímenes históricos y epistémicos, donde el despertar de tradiciones, la supervivencia de significados y la gestación de nuevos saberes se articulan con las ciencias y las tecnologías modernas; donde se abren las posibilidades para la convivencia de lo diverso. Estas son alternativas promisorias para un nuevo mundo, para un futuro sustentable. 


\section{A UN NIVEL LOCAL, PENSANDO DESDE LO GLOBAL}

Las intervenciones en comunidades y a nivel social, desde la perspectiva de la Psicología Social Comunitaria, están orientadas por las nociones de cambio social a través de la concientización y subsecuente participación de las comunidades en la solución de sus problemas. Este proceso requiere que la comunidad acceda a recursos materiales y psicológicos, y adquiera a través de ellos un mayor control sobre su ambiente.

Las problemáticas de desempleo, inseguridad, educación, gerenciamiento de organizaciones, trabajo en red etc. tienen la cualidad de anudar factores subjetivos (de pensamiento, sentimientos y acción), de pertenencias grupales, institucionales y pertenencias culturales específicas. Requieren por lo tanto de un abordaje interdisciplinario ya que exigen un profesional formado desde un marco referencial complejo que le permita un abordaje integrador de todas estas condicionantes variables en función de su potenciación.

Al igual que el abordaje a los problemas macro, es indispensable orientar a la Psicología Social a establecer un diálogo con los sujetos sociales, a la constitución de grupos de autoempleo para jóvenes, a la constitución y sostén de redes de organizaciones de la comunidad, el apoyo escolar a jóvenes de barrios pobres tendientes a la prosecución de sus estudios, capacitación informática, al abordaje de programas preventivos de adicciones, violencia, embarazo precoz, etc.

La integración social es central en términos de que la percepción de sí mismo, de los roles que se ejercen y la pertenencia grupal, se visualiza como un todo, y se adjudican en forma importante a dificultades de identidad, especialmente de integración de identidad, problemas sociales o parte de la explicación de la conducta de sujetos y grupos

Hablar de integración social es también reconocer la existencia de la diversidad, simultáneamente de las formas de relación entre grupos y categorías sociales, nos hemos referido a las culturas y grupos autóctonos, a los niños y jóvenes, pero también sería necesario referirse a las mujeres, La tendencia hacia una creciente preocupación por el proceso de formación de los sujetos sociales gira en torno con lo que se ha venido observando como un rasgo general de la investigación reciente sobre género: el mayor énfasis a los aspectos subjetivos y de formación de las identidades.

La constitución de los sujetos atañe también a los procesos de configuración de los significados sociales y su representación como ciudadana. Aunque promisoria, esta línea de análisis está aún por dar sus mejores frutos.

Las secuelas psicosociales de la violencia son efectos psicológicos y sociales producidos por el impacto de experiencias que no han podido ser procesadas y que tienen un carácter desestabilizador y perjudicial en la persona o grupo social (tomar en consideración la violencia política y sus secuelas psicosociales, stress postraumático en las zonas de sierra central del Perú), también pueden ser categorías para analizar al sector femenino.

La relación entre la representación de la ciudadanía y la confianza en la democracia es un tema que, en los últimos años, se ha tornado vigente y de preocupación de múltiples sectores de la sociedad, incluyendo las diversas disciplinas científico-sociales, los políticos y la propia sociedad civil en su conjunto. Pero la participación política no es sólo el canal 
de expresión de la ciudadanía, tal como se demuestra en una última investigación realizada por Arenas y cols., también se presentan espacios propios de la sociedad civil, de nivel regional y local.

Razones históricas que explican el porqué en un principio las mujeres fueron excluidas de la comunidad y el ámbito político. Durante buena parte del siglo XX, gracias a las luchas de las mujeres, la exclusión política, la desigualdad económica fundada en el género y la denigración cultural de lo femenino, se modifican y la ciudadanía femenina se va ampliando gradualmente. La inclusión del enfoque de género se concreta en las distintas convenciones y tratados internacionales que atacan la discriminación fundada en el sexo de las personas. La crítica a la oposición público-privada fue probablemente la primera gran consecuencia de la introducción de la perspectiva de género en el terreno de los estudios políticos y sobre la ciudadanía. Se destaca que la ideologización de la esfera privada como carente de sustrato político cumplía, entre otros, el cometido de marginar a las mujeres de este importante ámbito social y asegurar su control

\section{FORMAS NO INSTITUCIONALIZADAS DE ACCIÓN POLÍTICA}

La revalorización de las formas no institucionalizadas de acción política, alude a la recuperación de prácticas políticas femeninas por mucho tiempo invisibles, en parte por inadecuación con los marcos teóricos tradicionales, Una revisión que sobre el tema realizan a finales de la década de los ochenta, distingue tres tipos de movilizaciones con presencia femenina:

1. las que se conforman alrededor de las identidades, del género y la edad.

2. las que se estructuran alrededor de demandas específicas de servicios urbanos, casi siempre en sectores populares.

3. y las que lo hacen en torno a la defensa de la vida y los derechos humanos.

Las formas no institucionalizadas de acción política parecen recoger parte de lo que podría ser la especificidad de género de la movilización femenina: Sintomáticamente se configuran para la consecución de determinados objetivos prácticos, y una vez obtenidos, se evaporan. Rara vez es la acción política por sí misma el móvil que las impulsa. Según Foweraker (1998), la movilización política de las mujeres está profundamente enraizada en los roles femeninos tradicionales.

En la temática de la formación de las identidades (entre las que ubicamos la representación de ciudadanía) se ha pasado de una concepción centrada en la mujer y los roles y valores sociales adheridos a su condición, a otra en la que la individuación y los procesos de intersubjetividad se entienden más en su dimensión relacional y se destaca la mediación de ciertos aspectos socio-institucionales en su conformación

La dificultad reside en lograr una concepción positiva de la subjetividad femenina, en la que sobresalga la dimensión agente, activa, de su proceso de subjetivación, en oposición a una percepción pasiva y victimizante de la mujer, excesivamente dependiente y subsidiaria de las imágenes y figuras masculinas (Riquer, 1992). La conquista de la ciudadanía plena de las mujeres exige cambios tanto en la cultura como en el derecho con el fin de que 
en ambos campos se reflejen los principios de igualdad y libertad que deben regir los comportamientos y las garantías que ofrece un Estado democrático tanto a hombres como a mujeres.

Para lograr un salto cualitativo en la comprensión de las inequidades de género, se requiere de la realización de proyectos de mayor aliento que permitan, por un lado, articular el estudio de las inequidades de género con otras formas de inequidad social, y, por el otro, enmarcarlas en el contexto de las transformaciones acaecidas en la economía, el Estado y la sociedad civil

Como se trata además de un concepto multidimensional que articula aspectos socioestructurales y socio-simbólicos, las inequidades de género se manifiestan tanto en el acceso y control diferencial de recursos (físicos, socioeconómicos, socioculturales y políticos), como en las concepciones del mundo, el proceso de individuación y la construcción de identidades. Por lo anterior, surge como necesario incorporar en los análisis la temporalidad diferencial de las transformaciones en las distintas dimensiones, objetiva y subjetiva, en las prácticas y los discursos de los actores.

A pesar de los resultados, se notan cambios introducidos por la participación activa en la organización social en su inserción en el mapa de la ciudadanía por parte de las mujeres en el Perú. Frente a las inequidades de género, base en la que se presenta una débil confianza en la democracia y una limitada representación de su ciudadanía de las mujeres estudiadas en la investigación que comentamos, habría que plantearse instrumentos y conceptos superadores, desde y con ellas. Los conceptos de empoderamiento, igualdad, ciudadanía, podrían cumplir esta función. Tomemos como ejemplo el de empoderamiento.

El concepto de empoderamiento permite articular dimensiones sociales, familiares e individuales. En el nivel social supone: a) cambios en las leyes, derechos de propiedad y otras instituciones que reproducen la dominación masculina y de clase; b) el cuestionamiento de los valores e ideologías que legitiman la opresión femenina; c) el control de los recursos materiales (físicos, humanos y financieros), intelectuales (conocimientos, información e ideas), y de la ideología; d) la participación en las decisiones comunitarias y sociales, y e) la capacidad de organizarse y luchar en forma colectiva por sus derechos.

En los niveles individual y familiar: a) toma de consciencia del carácter injusto y antinatural de las diferentes formas de ordenamiento social, y de los derechos de igualdad, dignidad y justicia; b) la participación en las decisiones familiares; c) el cuestionamiento de las jerarquías existentes en la familia, y c) control sobre la propia vida (Sen y Grown, 1985; Batliwala, 1994). Es relevante examinar los factores que facilitan u obstaculizan una participación social efectiva que involucre: a) una real transferencia del poder de decisión hacia la comunidad; b) un involucramiento de diferentes sectores de la sociedad civil en el diseño e implementación de estrategias de desarrollo en el nivel nacional, y c) la ampliación de la ciudadanía social (Kliksberg, 1997; Elson, 1995).

Para terminar con el presente trabajo, queremos dejar reseñadas algunas reflexiones en torno a los retos que la Psicología Social tiene por delante, dentro de este plano asumir el principio de ubicarnos en los fenómenos emic de la realidad propia y local, desde la cotidianidad en la que se vive. 


\section{LÍNEAS DE INVESTIGACIÓN}

El estudio psicosociológico del trabajo y del desempleo. El análisis de temas como los significados del trabajo, la relación entre condiciones de trabajo y bienestar psicológico, incluyendo dentro de éste la satisfacción laboral y, finalmente, el estudio de los efectos psicosociales del desempleo.

Otra línea de investigación se inscribe dentro del área de la salud mental: el estudiar las causas psicosociales del deterioro psicológico, desde una perpectiva epidemiológica, analizar cómo las desigualdades sociales se traducen en desigualdades en el bienestar psicológico.

La idea que subyace a estos estudios es cómo la exclusión social que se produce como consecuencia del desempleo, por ejemplo, también da lugar a desigualdades en el plano de la salud mental. O cómo desigualdades en la estructura social, determinadas por la clase social o el género, por ejemplo, se traducen también en desigualdades en bienestar psicológico.

\section{CONCLUSIONES}

La investigación básica no tiene que ser irrelevante, es posible conciliar el interés teórico, el interés científico, el interés empírico con los intereses de la relevancia de la aplicación social.

Para ello se tiene que evitar el riesgo de psicologizar, sin embargo, es necesario que el subrayar el origen social de muchos problemas psicológicos no es incompatible con aplicar ciertas formas de intervención que pueden surgir desde la propia psicología y la psicología social en sus distintos ámbitos de aplicación.

La difusión del conocimiento que tenemos, de forma que éste llegue tanto a las instituciones con capacidad de intervención como a los propios desempleados. Junto con esto, no cabe duda de que el conocimiento que la psicología social tiene sobre este tema puede ser utilizado para diseñar programas de intervención encaminados a mejorar la situación psicológica y social de los desempleados

Se presenta como un reto el difundir a nivel popular los hallazgos de la ciencia psicológica en un lenguaje simple, a fin de que los periodistas, la gente, la opinión pública conozca estos trabajos científicos en un lenguaje comprensible que les permita efectivamente ver de qué manera son relevantes, por ejemplo trabajos de Milgram sobre obediencia (Julio F. Villegas, 1997), de formas de percibir la realidad, de la presencia y acción de los grupos y la cultura modeladora, etc.

Asumir la dialéctica integradora que supera la aproximación de sujeto versus contextos a sujetos en contextos, como una mirada de integración posible, es decir una psicología de sujetos en contextos (Becker, Morales y Aguilar, 1994) lo cual permite evitar caer en reduccionismos psíquicos o sociales

Aquí se presenta el reto de superar el asistencialismo-paternalismo y desarrollar el compromiso con el cambio social con las mayorías se hace más evidente poner en práctica lo sustentado en nuestro epígrafe que da sentido al título del presente trabajo. 


\section{REFERENCIAS BIBLIOGRÁFICAS}

1. P. Bourdieu y Loïc J. D. Wacquant (1995). Respuestas. México: Edit. Grijalbo.

2. Guzmán, Virginia (1998). "La equidad de género como tema de debate y de políticas públicas". En Eliana Largo (ed.) Género en el Estado. Estado del género. Ediciones de las mujeres 27. Santiago de Chile: Isis Internacional, pp. 55-70.

3. Hare-Mustin, Rachel y Janet Marecek (1994). Marcar la diferencia. Psicología y construcción de los sexos. Barcelona: Herder.

4. Kliksberg, Bernardo (1997). "Repensando el Estado para el desarrollo social: más allá de convencionalismos y dogmas". Revista Paraguaya de Sociología, año 34, N ${ }^{0}$ 99, mayo-agosto, pp. 159-197.

5. Sen, Amartya (1995). "Agency and Well-Being: the development agenda". En Noeleen Heyzer (ed.), A Commitment to the world's women. Perspectives on development for beijing and beyond. Nueva York: UNIFEM. 